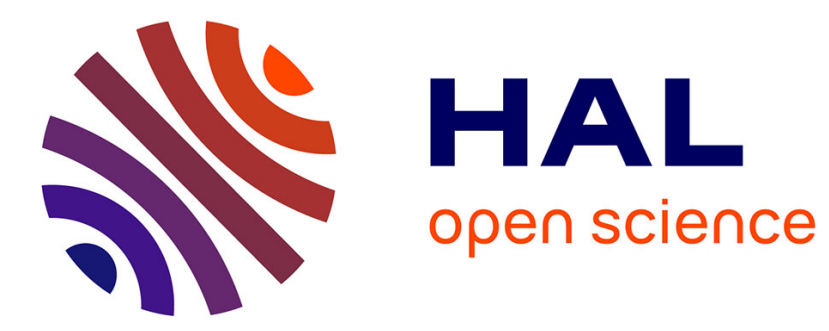

\title{
Transition from spherical cap to toroidal bubbles
}

Thomas Bonometti, Jacques Magnaudet

\section{- To cite this version:}

Thomas Bonometti, Jacques Magnaudet. Transition from spherical cap to toroidal bubbles. Physics of Fluids, 2006, vol. 18, pp. 1-12. 10.1063/1.2196451 . hal-00862651

\section{HAL Id: hal-00862651 \\ https://hal.science/hal-00862651}

Submitted on 17 Sep 2013

HAL is a multi-disciplinary open access archive for the deposit and dissemination of scientific research documents, whether they are published or not. The documents may come from teaching and research institutions in France or abroad, or from public or private research centers.
L'archive ouverte pluridisciplinaire HAL, est destinée au dépôt et à la diffusion de documents scientifiques de niveau recherche, publiés ou non, émanant des établissements d'enseignement et de recherche français ou étrangers, des laboratoires publics ou privés. 


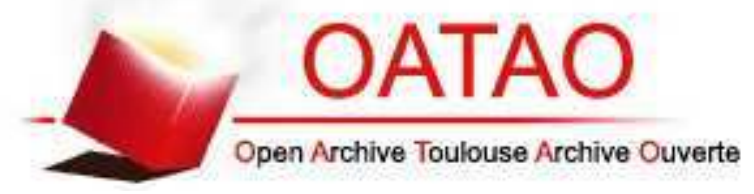

\section{Open Archive TOULOUSE Archive Ouverte (OATAO)}

OATAO is an open access repository that collects the work of Toulouse researchers and makes it freely available over the web where possible.

This is an author-deposited version published in : http://oatao.univ-toulouse.fr/ Eprints ID : 9405

To link to this article : DOI:10.1063/1.2196451

URL : http://dx.doi.org/10.1063/1.2196451

To cite this version : Bonometti, Thomas and Magnaudet, Jacques. Transition from spherical cap to toroidal bubbles. (2006). Physics of Fluids, vol. 18 ( $\left.\mathrm{n}^{\circ} 5\right)$. pp. 1-12. ISSN 1070-6631

Any correspondance concerning this service should be sent to the repository administrator: staff-oatao@ listes-diff.inp-toulouse.fr 


\title{
Transition from spherical cap to toroidal bubbles
}

\author{
Thomas Bonometti ${ }^{a)}$ and Jacques Magnaudet ${ }^{\text {b) }}$ \\ Institut de Mécanique des Fluides de Toulouse, UMR CNRS/INPT/UPS 5502, Allée Camille Soula, \\ 31400 Toulouse, France
}

Large gas bubbles rising under the effect of buoyancy are known to adopt either a spherical cap shape or to undergo a topological transition after which they become toroidal. We carry out an axisymmetric numerical investigation of the evolution of such large bubbles in the presence of both capillary and viscous effects. The numerical approach is of the volume of fluid type (it solves the Navier-Stokes equations on a fixed grid and transports the local volume fraction of one of the fluids), but does not involve any explicit reconstruction of the interface. The transition from spherical cap to toroidal bubbles is studied in the parameter space built on the Bond (Bo) and Archimedes (Ar) numbers, which compare the strength of inertial effects to that of capillary and viscous effects, respectively. Preliminary tests show that the position of this transition is very sensitive to the grid resolution; these tests are used to select grid characteristics that yield grid-independent results. Two markedly different transition scenarios, corresponding to the limit of large Ar and large Bo, respectively, are then identified. In the first case, the front of the bubble is pierced by an upward jet coming from the rear of the bubble. In contrast, in the limit of large Bo, a downward jet develops at the front part and pierces the rear of the bubble, unless viscous effects are sufficient to stabilize the front. We also determine the position of the transition for intermediate values of Bo and Ar and discuss the connection between present axisymmetric results and experimental situations in which the bubble is followed by a turbulent wake. We finally examine a puzzling feature of these large bubbles which is that, given an initial gas volume, the final bubble topology appears to depend dramatically on the initial conditions. Indeed, we find that initially oblate bubbles may result in stable spherical cap bubbles for values of Bo and Ar well beyond those for which initially spherical bubbles of similar volume undergo the topological transition. This remarkable influence of the initial shape is shown to be due to the influence of the oblateness on both the bubble acceleration and the hydrostatic pressure difference between the two bubble poles.

\section{INTRODUCTION}

Toroidal buoyancy-driven gas bubbles are fascinating objects frequently produced by divers and dolphins. ${ }^{1,2}$ They may also be easily generated in laboratory experiments or in swimming pools by opening and closing rapidly a valve closing a small vessel in which air has been previously stored. ${ }^{3}$ These large bubbles are remarkably stable and may rise over distances of several tens times their diameter. Small toroidal bubbles are also encountered as the result of the collapse of cavitation bubbles near a wall. ${ }^{4,5}$ Walters and Davidson ${ }^{3}$ carried out a detailed experimental study of the formation and evolution of toroidal buoyancy-driven bubbles rising in water. Their bubbles had volumes ranging from approximately 6 to $115 \mathrm{~cm}^{3}$. They also analyzed theoretically the first stages of the evolution of large, initially spherical bubbles by considering that the bubble shape evolution is driven by the competition of inertia and buoyancy only. This simple irrotational model shows that the rear part of the bubble, which experiences a (relatively) high pressure owing to the hydrostatic head, rises faster than the front, and comes in contact

\footnotetext{
${ }^{a)}$ Electronic mail: bonometti@imft.fr

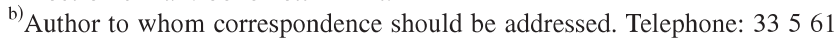
2858 07. Fax: 335612859 91. Electronic mail: magnau@imft.fr
}

with it at a finite time, leading eventually to the formation of a toroidal bubble. Walters and Davidson modeled these toroidal bubbles as hollow vortex rings rising in an irrotational inviscid environment, still disregarding surface tension effects. By monitoring the rise velocity and the ring and core diameters of the bubbles they produced, they were able to validate this simple model, showing that the circulation remains almost constant during the rise. Moreover, they obtained a reasonable estimate of this circulation by evaluating the velocity potential difference between the front and the rear of the initial bubble just before the pinch-off. A theoretical investigation of the evolution of a toroidal bubble in both inviscid and slightly viscous fluid was carried out by Pedley. ${ }^{6}$ His study shows in particular that, while viscous effects do not alter the evolution of the ring radius (which grows as the square root of time $t$ to satisfy the conservation of the vertical impulse), they slightly reduce the rise velocity which decays as $t^{1 / 2}$.

The first detailed numerical investigation of the formation and evolution of toroidal bubbles in an inviscid fluid with nonzero surface tension was achieved by Lundgren and Mansour $^{1}$ using the boundary element method. Since their technique could not deal with the topological change right at the time of the pinch-off, they had to compute separately the 
simply- and doubly-connected stages of the bubble evolution. They were able to determine the critical size beyond which the bubble becomes toroidal and the corresponding pinch-off time. Then, they studied the toroidal stage by determining the circulation in the same manner as Walters and Davidson and by considering several arbitrary values of the initial ratio between the core and ring diameters. This enabled them to examine the evolution of the ring diameter and the deformation of the core cross section as a function of the bubble aspect ratio. In agreement with theoretical predictions, ${ }^{6,7}$ they found the ring diameter to evolve essentially linearly with the vertical position of the bubble. However, they also pointed out the presence of small-amplitude high-frequency oscillations of the ring diameter and rise velocity, a phenomenon due to the combined effect of the added-mass force and the Kutta-Joukowski lift force.

During the last decade, computational techniques capable of dealing directly with free-surface flows in the presence of viscous effects and topological changes have become available. Sussman and Smereka ${ }^{8}$ employed a level set technique to study various axisymmetric two-phase flows with large density contrasts. Regarding the formation of toroidal bubbles, they also used in parallel a boundary element technique very similar to that of Lundgren and Mansour, but with some additional numerical "surgery" making it able to overcome the pinch-off singularity. They essentially concluded that the two numerical approaches yield results in close agreement up to the pinch-off time. However, they also noticed that significant differences, which develop from the pinch-off region, take place in the subsequent evolution of the toroidal bubble shape. A more detailed numerical investigation was performed by Chen et al. ${ }^{9}$ using the volume of fluid approach. They explored separately the role of surface tension, viscosity, and density ratio on the evolution of the bubble geometry. As may be expected, they observed that, when surface tension or liquid viscosity is increased beyond a certain value, the bubble geometry remains simply connected, the final bubble taking a spherical cap shape. They also noticed that a toroidal bubble rises more slowly than its spherical cap counterpart, an effect of the circulation around the toroidal bubble which slows down its upward motion.

While the mechanisms governing the formation of toroidal bubbles and the qualitative influence of the various physical parameters on their evolution have been clarified in the above studies, the precise localization of the transition from spherical cap to toroidal bubbles in the appropriate parameter space has not been achieved in the presence of finite viscous effects. Also, a puzzling, still poorly understood feature of large buoyancy-driven bubbles is that, depending on the geometry of the injection system used to generate them, they seem to be able to evolve either towards a spherical cap shape or to become toroidal. ${ }^{11}$ For instance, while Walters and Davidson ${ }^{3}$ observed toroidal air bubbles whose volume ranged from 6 to $115 \mathrm{~cm}^{3}$, Davies and Taylor ${ }^{10}$ were able to produce spherical cap bubbles with a volume up to $200 \mathrm{~cm}^{3}$.

The formation and dynamics of spherical cap bubbles are better understood and have been the subject of detailed studies. ${ }^{11-14}$ However, an intriguing question is the existence of very large spherical cap bubbles followed by a turbulent wake. ${ }^{15,16}$ Computer limitations still prevent reliable simulations of such situations in which the flow is threedimensional and time-dependent; the shape of the bubble evolves in time and its path is not strictly rectilinear. However, some insight into the mechanisms that contribute to ensure the stability of these large bubbles in the presence of small-scale turbulence may perhaps be gained by analyzing the effects of viscosity on the flow at the back of the bubble and making use of the concept of eddy viscosity.

The above points are the central subject of the present numerical investigation. The method employed here, briefly described in Sec. II, borrows some features from the volume of fluid approach (since it is essentially based on the transport of the volume fraction of one of the fluids) as well as to the level set technique (as no explicit reconstruction of the interface is carried out). Validations focused on bubble evolution in contrasted flow regimes are provided in Sec. III; the influence of the spatial resolution on the evolution of large spherical cap/toroidal bubbles is also investigated. Then, accurate axisymmetric computations involving larger computational domains and finer grid resolutions than those used in previous studies (especially in Ref. 9) are performed and discussed in Sec. IV to determine properly the topological transition as a function of the Bond and Archimedes numbers which compare the magnitude of inertial effects to that of surface tension effects and viscous effects, respectively. Finally, in Sec. V we investigate the influence of the initial bubble shape on the evolution of its subsequent topology and show that, compared to initially spherical bubbles, bubbles with an initial oblateness are able to remain simplyconnected over a much larger parameter range.

\section{NUMERICAL APPROACH}

We assume the suspending liquid and the gas filling the bubble to be both Newtonian and incompressible, with uniform surface tension. The evolution of the two-phase flow is then classically described using the one-fluid formulation of the Navier-Stokes equations, viz.,

$$
\begin{aligned}
\frac{\partial \boldsymbol{V}}{\partial t}+\boldsymbol{V} \cdot \nabla \boldsymbol{V}= & -\frac{1}{\rho} \nabla P+\boldsymbol{g}+\frac{1}{\rho} \nabla \cdot\left[\mu\left(\nabla \boldsymbol{V}+{ }^{t} \nabla \boldsymbol{V}\right)\right] \\
& -\frac{\sigma}{\rho}(\nabla \cdot \boldsymbol{n}) \boldsymbol{n} \delta_{I} ; \quad \nabla \cdot \boldsymbol{V}=0,
\end{aligned}
$$

where $\boldsymbol{V}, P, \rho$, and $\mu$ are, respectively, the local velocity, pressure, density, and dynamical viscosity in the flow, $g$ denotes gravity, and $\sigma$ is the surface tension. The surface delta function $\delta_{I}$ is zero outside the interface, the unit normal of which (directed toward the liquid) is denoted by $\boldsymbol{n}$. The local volume fraction of gas obeys

$$
\frac{\partial C}{\partial t}+\boldsymbol{V} \cdot \nabla C=0
$$

This volume fraction equals 1 (respectively, zero) in cells filled with gas (respectively, liquid) and takes intermediate values in cells belonging to the transition region, the 
thickness of which will be specified below. The local density and dynamical viscosity are evaluated using a linear interpolation law, namely

$$
\rho=C \rho_{g}+(1-C) \rho_{l} ; \quad \mu=C \mu_{g}+(1-C) \mu_{l},
$$

where the subscripts $g$ and $l$ refer to the gas and liquid, respectively. The capillary force is transformed into a volume force using the continuum surface force model proposed by Brackbill et al. ${ }^{17}$ Hence, we write

$$
\frac{\sigma}{\rho}(\nabla \cdot \boldsymbol{n}) \boldsymbol{n} \delta_{I}=\frac{\sigma}{\rho} \nabla \cdot\left(\frac{\nabla C}{\|\nabla C\|}\right) \nabla C .
$$

The Navier-Stokes equations (1) and (2) are solved using the JADIM code developed in our group. Details on the spatial discretization and time-advancement algorithm used in this code for constant density situations may be found in several previous publications. ${ }^{18-20}$ Briefly, the momentum equations are discretized on a staggered grid using a finite volume approach. The spatial discretization is performed using secondorder centered differences. Time advancement is achieved through a third-order Runge-Kutta method for advective and source terms and a Crank-Nicolson method for viscous stresses. Incompressibility is satisfied at the end of each time step through a projection method. The overall algorithm is second-order accurate in both time and space.

The main changes introduced in this procedure by the two-phase nature of the flows considered here are related to the treatment of Eq. (2) [which drives the variable density and viscosity in (1)] and to the capillary force. To solve Eq. (2), we split it into successive one-dimensional steps along each grid direction $i(i=1,3)$ and use a Zalesak scheme ${ }^{21}$ during each substep. This flux-limiting procedure, which combines the use of a low-order (here, first-order) and highorder (here, eighth-order) approximation of the advective flux in (2) guarantees the positivity and monotonicity of $C$ with second-order spatial accuracy. To prevent the transition region $0<C<1$ from thickening as time proceeds, we make the velocity field involved in (2) locally constant across this region. To this end, instead of advecting $C$ with the actual velocity $V_{i}(\boldsymbol{x}, t)$ at points $\boldsymbol{x}$ located within the transition region, we advect it with the velocity $V_{i}\left(\boldsymbol{x}_{0}, t\right), \boldsymbol{x}_{0}$ being the point closest to $x$ that lies on the iso- $C$ line $C=0.5$ and is located either on the $i$ th grid line or on the streamline passing through $\boldsymbol{x}$, depending on which of them makes the smallest angle with the local volume fraction gradient. With the above procedure, the thickness of the transition region is maintained within three cells, except in some regions of very large curvature. The modified velocity field is only used for the computation of $C$, while the actual velocity field $\boldsymbol{V}(\boldsymbol{x}, t)$ remains the only one involved in the Navier-Stokes equations (1). Nevertheless, the above procedure and the onedimensional splitting of (2) results in small mass errors that may accumulate as time proceeds. Therefore, a final modification of the local volume fraction $C$ is performed within the transition region, so as to maintain the variation of the global mass in each fluid below a given percentage of the initial mass (generally $0.1 \%$ ), while keeping $C$ between 0 and 1 everywhere. This correction results in a stiffening of the tran- sition region and a slight displacement of the position of the iso- $C$ line $C=0.5$ within it. Further details about the transport strategy for $C$ may be found in Ref. 22 .

The time-advancement procedure of the coupled system (1)-(3) starts by solving (2), prior to solving the momentum equation. Hence, starting from $C^{n}(x)$ and $\boldsymbol{V}^{n}(x)$ at time $n \Delta t$ ( $\Delta t$ being the time step), we first obtain the solution $C^{n+1}(x)$ corresponding to time $(n+1) \Delta t$ using the technique described above, and use it to evaluate the density $\rho^{n+1}(\boldsymbol{x})$ and the viscosity $\mu^{n+1}(\boldsymbol{x})$ through (3). Then, we define the second-order approximations of $\rho$ and $\mu$ at time $(n+1 / 2) \Delta t$ as $\rho^{n+1 / 2}=\left(\rho^{n}+\rho^{n+1}\right) / 2, \mu^{n+1 / 2}=\left(\mu^{n}+\mu^{n+1}\right) / 2$ and use them throughout the time step $[n \Delta t,(n+1) \Delta t]$ to solve (1) and obtain a provisional velocity field $\boldsymbol{u}^{*}$. As the volume fraction is defined at pressure nodes, linear interpolations are used to obtain the density and viscosity at velocity nodes. Finally, the variable density projection method by which the final velocity field $\boldsymbol{u}^{n+1}$ is made divergence-free requires the solution of a pseudo-Poisson equation of the form $\nabla \cdot(\nabla \phi / \rho)$ $=\nabla \cdot \boldsymbol{u}^{*}$ for the pressure increment $\phi$. The corresponding linear system is solved by a Jacobi preconditioned conjugate gradient technique (JCG routine of the ITPACK Library).

The capillary term (4) is evaluated by approximating $C$ at time $(n+1 / 2) \Delta t$ as $C^{n+1 / 2}=\left(C^{n}+C^{n+1}\right) / 2$. To obtain the volume integral of the right-hand side of (4) over a computational cell bounded by a surface $\Gamma$ with a unit normal $N$, we rewrite this term in the form $\sigma\left\{\int_{\Gamma} \boldsymbol{N} \cdot(\nabla C /\|\nabla C\|) d \Gamma\right\} \overline{\nabla C / \rho}$, where $\overline{\nabla C / \rho}$ is a volume average of $\nabla C / \rho$ over the cell, all contributions being evaluated at velocity nodes using centered differences and secondorder interpolations. As is well known, the sharp variations of $C$ throughout the transition region tend to generate spurious peaks in the curvature and lead to a poor evaluation of the capillary force. To avoid such artifacts, the capillary force is calculated using a smoothed distribution $\hat{C}$ of the volume fraction obtained from $C$ through several successive applications of a weighted average over the surrounding grid points. In two dimensions, the elementary smoothing procedure around a given grid point $(i, j)$ takes the form $\hat{C}_{i, j}=3 / 4 C_{i, j}$ $+\left(C_{i+1, j}+C_{i-1, j}+C_{i, j+1}+C_{i, j-1}\right) / 16$.

Extensive tests of the code based on the above method were carried out and may be found in Ref. 22. Tests concerned with the computation of capillary-gravity waves show in particular that the spatial accuracy of the overall method lies between first and second order, even though the initial wave amplitude is much less than the grid spacing.

\section{PRELIMINARY TESTS WITH RISING BUBBLES}

Before we apply the above method to discuss the formation of toroidal bubbles, we consider some extra test cases with a double goal. First, we wish to confirm that our code properly captures the dynamics of buoyancy-driven bubbles in contrasted well-documented regimes. Second, we need to determine the grid characteristics required to obtain gridindependent solutions in the regime where the transition from spherical cap to toroidal bubbles is likely to occur.

For given density and viscosity ratios, the dynamics of isolated bubbles may be characterized using the rise Rey- 


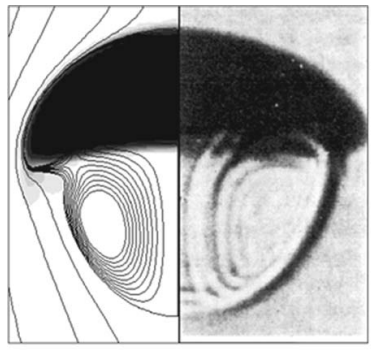

(a)

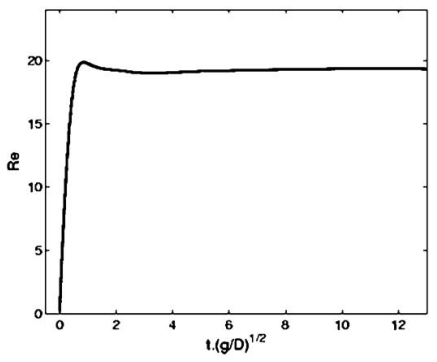

(b)
FIG. 1. (a) Comparison of the computed bubble shape and streamlines (left) with those reported in Fig. 1(a) of Hnat and Buckmaster (Ref. 13) (right) $\left(\rho_{l} / \rho_{g}=10^{3}, \mu_{l} / \mu_{g}=10^{2}, \mathrm{Mo}=0.065, \mathrm{Bo}=39.6\right)$. The black zone on the left part corresponds to cells with $C>0.99$, whereas the white zone corresponds to cells with $C<0.01$. (b) Time evolution of the bubble Reynolds number. The final Reynolds number is $\operatorname{Re} \approx 19.3$.

nolds number $\operatorname{Re}=\rho_{l} U D / \mu_{l}$ (which is an output of the experiment), the Bond (or Eötvos) number $\mathrm{Bo}=\rho_{l} g D^{2} / \sigma$ and the Morton number $\mathrm{Mo}=g \mu_{l}^{4} / \rho_{l} \sigma^{3}, D$ being the equivalent diameter of the bubble [equivalently we could use the Kapitza number $\mathrm{Ka}=\mathrm{Mo}^{-1 / 6}$, which is the ratio of the viscous length scale $l_{\nu}=\left(\mu_{l}^{2} / \rho_{l}^{2} g\right)^{1 / 3}$ over the capillary length scale $\left.l_{c}=\left(\sigma / \rho_{l} g\right)^{1 / 2}\right]$. The rise velocity $U$ of the bubble centroid is defined through

$$
U(t)=\frac{\int_{\vartheta} C(\boldsymbol{x}, t) \boldsymbol{V}(\boldsymbol{x}, t) \cdot \boldsymbol{e}_{z} d \vartheta}{\int_{\vartheta} C(\boldsymbol{x}, t) d \vartheta},
$$

where $C$ is the gas volume fraction, $\boldsymbol{e}_{z}$ is the unit vector in the vertical direction, and $\vartheta$ denotes the volume of the whole computational domain. The computations are axisymmetric and are performed within a cylindrical $(r, z)$ domain $5.5 D$ $\times 12.6 D$ large. We use a $140 \times 700$ grid size with a regular spacing in the $z$ direction $\left(\Delta z / D=1.8 \times 10^{-2}\right.$, corresponding to 55 grid cells per bubble diameter). In the $r$ direction, a similar regular spacing $\left(\Delta r / D=1.8 \times 10^{-2}\right)$ is used over a central zone of radius $D$ corresponding to the region crossed by the bubble and that of maximum liquid entrainment, while larger cells are used beside this region, following an arithmetic distribution. Free-slip boundary conditions are imposed on the top, bottom, and lateral boundaries, so that the fluid entrained upward by the bubble slowly goes down near the lateral boundary; obviously computations are stopped before the bubble comes close to the upper boundary, to avoid contamination of the results by confinement effects.

We first select the physical parameters so as to obtain a Bond number $\mathrm{Bo}=39.6$ and a Morton number $\mathrm{Mo}=0.065$, as in Fig. 1(a) of Hnat and Buckmaster. ${ }^{13}$ The density and viscosity ratios of the two fluids are set to $10^{3}$ and $10^{2}$, respectively. The final bubble shape and near-wake structure, reached after a time of $O\left(\rho_{l} D^{2} / \mu_{l}\right)$, are shown in Fig. 1(a). The agreement with the original photograph of Hnat and Buckmaster $^{13}$ is excellent. Figure 1(b) shows the evolution of the bubble Reynolds number as the bubble rises; the final Reynolds number is 19.3. A second computation with the lateral boundary located $10.5 \mathrm{D}$ away from the symmetry axis was also carried out and yielded a final Reynolds number of 19.9. Both values agree within $2 \%$ with the experimental value of 19.6 and indicate that the initial computational domain induces little artificial confinement.

As a second example we consider a slightly deforming bubble rising at higher Reynolds number. For this purpose, we select $\mathrm{Mo}=5 \times 10^{-8}, \mathrm{Bo}=1$, and keep the density and viscosity ratios unchanged. To study this situation, we extend the numerical domain in the vertical direction, since the bubble requires a longer distance to reach its final speed. The corresponding grid is made of $140 \times 900$ cells in the $(r, z)$ directions. The final shape of the bubble shown in Fig. 2(a) (left) is close to an oblate spheroid of aspect ratio 1.42. The evolution of the Reynolds number, displayed in Fig. 2(b), yields a final value of 101.6. As the Weber number We $=\rho_{l} U^{2} D / \sigma$ may be expressed as $\mathrm{We}=\operatorname{Re}^{2}(\mathrm{Mo} / \mathrm{Bo})^{1 / 2}$, we have $\mathrm{We} \approx 2.31$. Detailed experiments in pure water ${ }^{23}$ indicate that the aspect ratio of an air bubble having this Weber number is 1.43 , in excellent agreement with the numerical prediction. We also run the same case using the single-phase boundary-fitted version of the JADIM code, ${ }^{18-20}$ which makes use of the same Navier-Stokes solver as the present interface-capturing version but employs an orthogonal grid to map exactly the bubble shape, allowing the exact boundary conditions to be applied right at the bubble surface. ${ }^{24,25}$

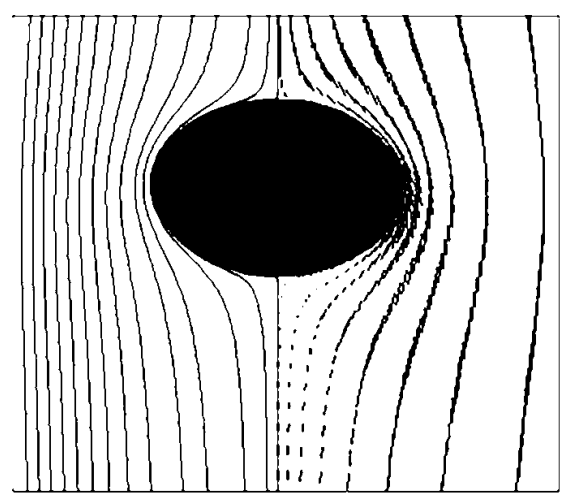

(a)

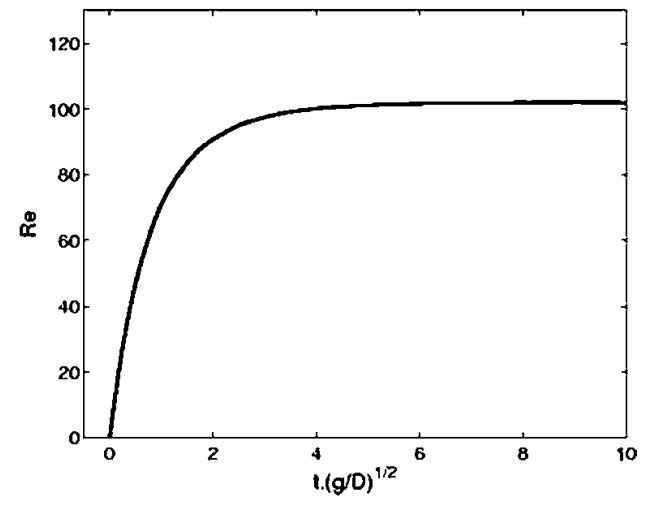

(b)
FIG. 2. (a) Left: bubble shape and streamlines obtained using the present method for $\rho_{l} / \rho_{g}=10^{3}, \mu_{l} / \mu_{g}=10^{2}$, $\mathrm{Mo}=5 \times 10^{-8}, \mathrm{Bo}=1$; the final aspect ratio of the bubble is 1.42 . Right: streamlines obtained with the boundary-fitted method around a spheroidal bubble with an aspect ratio 1.42 . The black zone on the left part corresponds to cells with $C>0.99$, whereas the white zone corresponds to cells with $C<0.01$. (b) Time evolution of the bubble Reynolds number. The final Reynolds number is $\mathrm{Re} \approx 101.6$. 
(a)

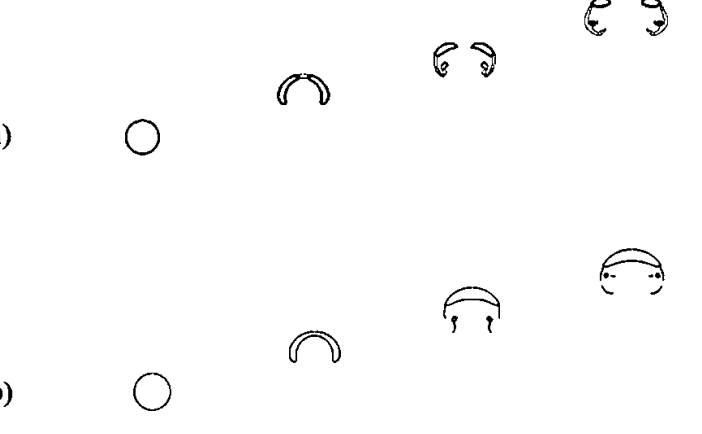

FIG. 3. Evolution of a bubble corresponding to $\rho_{l} / \rho_{g}=10^{3}, \mu_{l} / \mu_{g}=10^{2}$, $\mathrm{Bo}=10^{2}, \mathrm{Mo}=10^{-3}$. (a) $\Delta r / D=1 / 55$; (b) $\Delta r / D=1 / 110$. Time between successive views is $2.21(\mathrm{~g} / D)^{1 / 2}$; iso- $C=0.01,0.5$, and 0.99 .

Here, we use this boundary-fitted version to obtain the final drag force, assuming that the bubble stays fixed in a uniform stream of velocity $V_{0}$ far upstream and keeps a frozen spheroidal shape of aspect ratio 1.42 (i.e., we do not consider the normal stress balance at the bubble surface). For a given Reynolds number $\operatorname{Re}_{0}=\rho_{l} V_{0} D / \mu_{l}$, this methodology yields a drag force $F_{D}=\rho_{l} \pi D^{2} C_{D}^{B F} V_{0}^{2} / 8$, from which the drag coefficient $C_{D}^{B F}\left(\mathrm{Re}_{0}\right)$ is obtained. On the other hand, the present interface-capturing method provides a final rise velocity $U$ and a drag coefficient $C_{D}$ resulting from a balance between the buoyancy force and the drag force, viz., $C_{D}=4 g(1$ $\left.-\rho_{g} / \rho_{l}\right) D / 3 U^{2}$. We find $C_{D}=0.572$, in excellent agreement with the value $C_{D}^{B F}\left(\operatorname{Re}_{0}=101.6\right)=0.576$ provided by the boundary-fitted computation. This agreement extends beyond global parameters, as Fig. 2(a) indicates that both methods provide very similar streamline patterns. The above two tests show that the present method, combined with a grid resolution about 55 cells per bubble diameter, yields accurate results for deformable bubbles with Reynolds number of $O(10)$ to $O\left(10^{2}\right)$.

Finally, we compute the evolution of a bubble corresponding to $\rho_{l} / \rho_{g}=10^{3}, \mu_{l} / \mu_{g}=10^{2}, \mathrm{Bo}=10^{2}, \mathrm{Mo}=10^{-3}$ with the grid used in the first example above. Figure 3(a) shows that the rear part of the bubble quickly comes in contact with its front. The resulting thin layer of gas then pinches off and yields the formation of a toroidal bubble followed by a long skirt. Later this skirt rolls up on itself and detaches from the torus, forming a second toroidal ring. To examine the influence of the spatial resolution in this regime, we doubled it (i.e., we used $\Delta r / D \approx 1 / 110$ for $r \leqslant D$ and $\Delta z / D \approx 1 / 110$ ) and recomputed the same case. The resulting evolution is shown in Fig. 3(b). No pinch-off is now noticed. Instead, the bubble evolves towards a spherical cap shape with a short skirt. Therefore, we have to conclude that the toroidal bubble predicted in Fig. 3(a) is an artifact due to a somewhat inadequate resolution. Grid resolution is of course a common issue in all problems involving topological changes, such as breakup and coalescence, as the gas or liquid film always ruptures when its thickness becomes of the order of a grid cell. The question is then, for a given physical problem and a given range of physical parameters, to determine the minimum grid resolution beyond which the results become grid independent in a macroscopic sense, i.e., the final interface geometry, the breakup or coalescence time, and the global dynamics do not vary any more with the grid resolution. In the present case, based on several examples in the same flow regime, resolutions ranging from 55 to 170 cells in the region $r \leqslant D$ were used in preliminary tests. These tests revealed that the results become grid independent when the number of cells in this central region exceeds 100 . This is especially the case of computations reported in Secs. IV A and IV B, which were also run on grids with 170 cells per bubble diameter. Having established this, the computations that led to the results described below were all run on a grid with 111 uniform cells in the region $r \leqslant D$. Note the difference between present grid requirements imposed by the topological change and conclusions of the previous tests, which indicate that the other stages of bubble dynamics are properly captured with a twice coarser grid. The computational domain extends up to $5.5 D$ (respectively, $6.3 D$ ) in the radial (respectively, vertical) direction to avoid confinement effects. The outer region $D$ $<r \leqslant 5.5 D$ is discretized with 29 cells whose size increases with $r$ following an arithmetic distribution. In the vertical direction, the grid is uniform and still comprises 700 cells, i.e., the grid spacing $\Delta z / D$ is about $1 / 111$. Note that with the above choice, the grid cells crossed by the bubble have a squared cross section.

\section{THE TRANSITION FROM ELLIPSOIDAL CAP BUBBLES TO TOROIDAL BUBBLES}

We are now in position to deal with the range of parameters where the formation of toroidal bubbles is likely to occur. Throughout this section, the density and viscosity ratios are set to $10^{3}$ and $10^{2}$, respectively. We only vary the Bond number $\mathrm{Bo}=\rho_{l} g D^{2} / \sigma$ and the Archimedes number $\mathrm{Ar}$ $=\rho_{l} g^{1 / 2} D^{3 / 2} / \mu_{l}$ which may be thought off as the Weber and Reynolds numbers based on the gravitational velocity $(g D)^{1 / 2}$, respectively [strictly speaking the relevant velocity scale is $\left(1-\rho_{g} / \rho_{l}\right)(g D)^{1 / 2}$ but the selected density ratio makes the effect of the gas density negligible]. All computations presented in this section are axisymmetric and start from rest with a spherical bubble shape.

\section{A. High-Ar regime}

We first consider the regime where viscous effects are weak. Hence, we choose the physical parameters so that the final bubble Reynolds number is of $O\left(10^{3}\right)$, which is expected to correspond to values of the Archimedes number about $1.5 \times 10^{3}$. Keeping $\mathrm{Ar}$ constant, we vary the Bond number from low values $(\mathrm{Bo} \sim 1)$ to high values $\left(\mathrm{Bo} \sim 10^{3}\right)$ and detect the range within which the topological transition takes place. Figure 4 shows the evolution of two bubbles corresponding to $\mathrm{Bo}=32$ and $\mathrm{Bo}=35$, respectively, with $\mathrm{Ar}$ $=1.4 \times 10^{3}$ in both cases. The two bubbles first strongly deform due to the extra pressure provided by the hydrostatic head $\rho_{l} g D$ in the rear-pole region. The pressure difference between the two poles generates a liquid jet which quickly reduces the vertical diameter of the bubble. However, for $\mathrm{Bo}=32$, surface tension is still strong enough to limit the curvature of the apex of the jet and to slow down its upward motion in a way that preserves a simply-connected shape. 


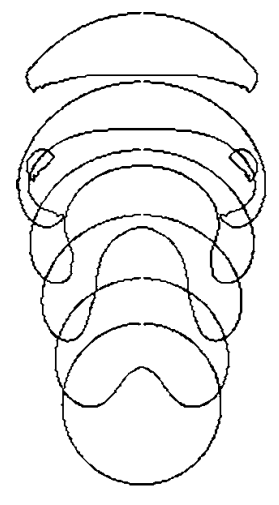

(a)

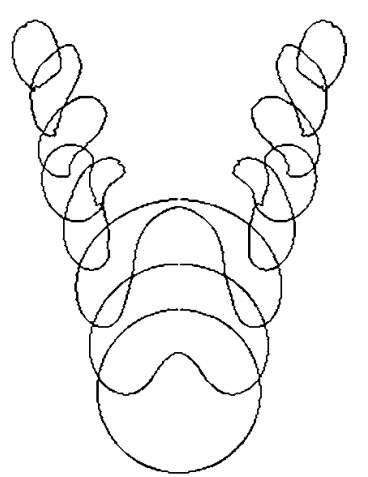

(b)
FIG. 4. Evolution of two high-Re bubbles $\left(\mathrm{Ar}=1.4 \times 10^{3}\right)$ with slightly different Bond numbers. (a) $\mathrm{Bo}=32$. (b) $\mathrm{Bo}=35$. The time interval between successive views is $\Delta \tau=0.664\left[\tau=t(g / D)^{1 / 2}\right]$.

The bubble then eventually reaches a skirted spherical cap shape. The situation is dramatically different in the second case [Fig. 4(b)] where the curvature in the top region of the jet is slightly larger, owing to the somewhat smaller surface tension. The bubble then pinches off along its vertical diameter at time $\tau \approx 1.37$ [with $\tau=t(g / D)^{1 / 2}$ ] and becomes toroidal. Since the bubble has a large thick skirt just before the pinch-off, its cross section is far from circular and rather takes a kidney-like shape just after the topological change. Surface tension effects then make it retract to become more circular and large shape oscillations take place. The radial expansion of the toroidal bubble required to preserve momentum conservation ${ }^{1,6}$ is clearly visible in Fig. 4(b).

Further computations performed for $\mathrm{Bo}<32$ at both higher and lower values of Ar confirm that the bubble remains simply-connected in all cases. Hence, we conclude that the transition from spherical cap bubbles to toroidal bubbles takes place in the range $32 \leqslant \mathrm{Bo} \leqslant 35$ when viscous effects are negligibly small, which corresponds to $2.1 \mathrm{~cm}^{3}$ bubbles for water under standard conditions. This conclusion is in full agreement with that obtained through boundary element computations by Lundgren and Mansour, ${ }^{1}$ who considered the inviscid limit $(\mathrm{Ar}=\infty)$ and found the topological change to occur in the range $32 \leqslant \mathrm{Bo} \leqslant 40$. The shape evolution displayed for both $\mathrm{Bo}=32$ and $\mathrm{Bo}=40$ in their Figs. 2(d) and 2(e) and reprinted here as Fig. 5 reveals excellent agreement with present results up to pinch-off (since their numerical method could not handle the topological change, the first part of their computations stopped just before the singularity occurred). Note that Lundgren and Mansour normalized their time $T$ by the initial bubble radius, so that $T$ has to be divided by $2^{1 / 2}$ to be compared with the times $\tau$ of our snapshots in Fig. 4. With this renormalization, Figs. 4(a) and 5(b) reveal an excellent agreement on both the shape and the position of the bubble for a given $\tau$. Moreover the pinch-off time found by Lundgren and Mansour for $\mathrm{Bo}=40$ becomes 1.26 , which is slightly less than the value of 1.37 found here for Bo $=35$, a difference to be expected since the pinch-off time increases as Bo decreases towards its critical value.

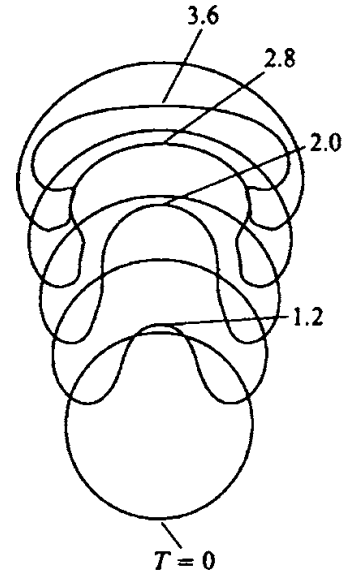

(a)

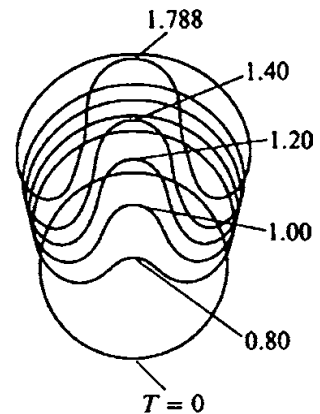

(b)
FIG. 5. Lundgren and Mansour's figures [Figs. 2(d) and 2(e)] showing the evolution of two bubbles with slightly different Bond numbers and infinite Archimedes number. Left: $\mathrm{Bo}=32$; right: $\mathrm{Bo}=40$.

Figure 6 shows the evolution of the bubble velocity for the two cases computed above. After an initial transient during which the bubble accelerates at a rate $d U / d t \approx 2 g$ (corresponding to an approximate balance between the addedmass force on the initial hollow sphere and the buoyancy force), the acceleration of its front strongly decreases due to viscous effects. Nevertheless, in the case $\mathrm{Bo}=32$, the front velocity of the bubble keeps on increasing slightly until $\tau$ $\approx 2.5$. In later stages this velocity stabilizes itself at a value close to $0.64(g D)^{1 / 2}$. According to the final snapshot in Fig. 4(a), the final radius of curvature $R_{c}$ of the corresponding spherical cap bubble is about $0.90 D$, so that we have $U$ $\approx 0.67\left(g R_{c}\right)^{1 / 2}$, in excellent agreement with the theoretical prediction of Davies and Taylor ${ }^{10} U=2 / 3\left(g R_{c}\right)^{1 / 2}$. The evolution of the centroid velocity is more complex because it also reflects the changes of shape of the bubble. For instance the centroid velocity exceeds the front velocity for $\tau \approx 0.5$

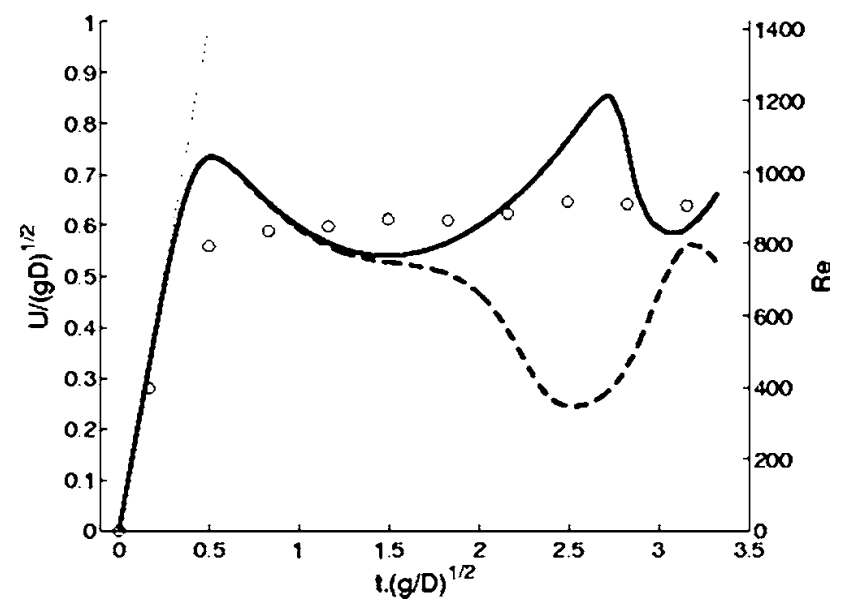

FIG. 6. Evolution of the bubble velocity and Reynolds number for two nearby Bond numbers at $\mathrm{Ar}=1.4 \times 10^{3}$. Open circles: front velocity for Bo $=32$; Solid line: centroid velocity for $\mathrm{Bo}=32$; dashed line: centroid velocity for $\mathrm{Bo}=35$; dotted line: $U=2 g t$. 


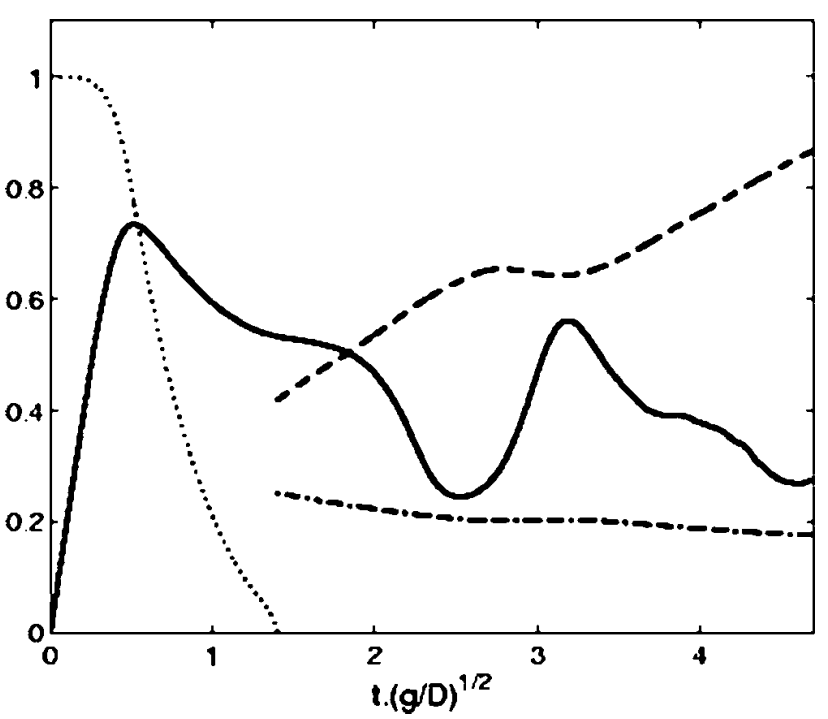

FIG. 7. Evolution of the bubble velocity (-), thickness of the vertical diameter (...), ring radius (---), and core radius (-.-.) for $\mathrm{Bo}=35, \mathrm{Ar}=1.4 \times 10^{3}$. The velocity and lengths are scaled by $(g / D)^{1 / 2}$ and $D$, respectively.

because the liquid jet forms at the rear and makes the gas located near the symmetry axis in the rear part of the bubble move faster than the front. Similarly, for $\mathrm{Bo}=32$, a significant part of the gas located in the skirt at time $\tau \approx 2.0$ then moves up towards the central part of the bubble which thickens, thus resulting in a strong increase of the centroid velocity up to $\tau \approx 2.7$. Slightly later, the thin curved skirt breaks up and is entrained in the wake, making the centroid velocity decrease sharply for $2.7 \leqslant \tau \leqslant 3.2$. In contrast, the centroid velocity of the toroidal bubble decreases by more than $50 \%$ for $1.8 \leqslant t \leqslant 2.5$ owing to the radial expansion of the ring (Fig. 7), whereas it reincreases by the same amount for 2.7 $\leqslant \tau \leqslant 3.2$, a stage during which the ring diameter decreases. The evolution of the bubble geometry after it becomes toroidal may also be appreciated from Fig. 7, where the radii of both the ring and the core are plotted (the ring radius $R_{r}$ is computed as $R_{r}(t)=\int_{S} C(\boldsymbol{x}, t) r d S / \int_{S} C(\boldsymbol{x}, t) d S$, where $S$ denotes the cross section of the computational domain, while the core radius $a$ is obtained by equating the bubble volume to $2 \pi^{2} R_{r} a^{2}$ ). Initially this aspect ratio has a quite small value about 1.7 and increases up to 4.8 at the point where the computation stops.

\section{B. High-Bond number viscous regime}

We now set surface tension to zero and seek the critical Archimedes number at which the transition to a toroidal shape occurs. Figure 8 shows the shape evolution of two different bubbles whose Archimedes numbers are $\mathrm{Ar}=79$ and 84 , respectively. In the first case, a liquid tongue develops and quickly broadens while the front part of the bubble flattens. The thickness of the bubble along its vertical axis then becomes very small. However, pinch-off does not occur because a large fraction of the liquid previously located in the external part of the bubble flows towards the center, allowing the central part to thicken again (Fig. 9). At the same time, the curvature of the external part becomes very large near the

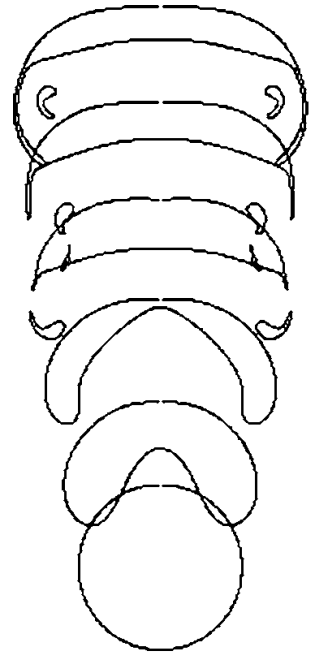

(a)

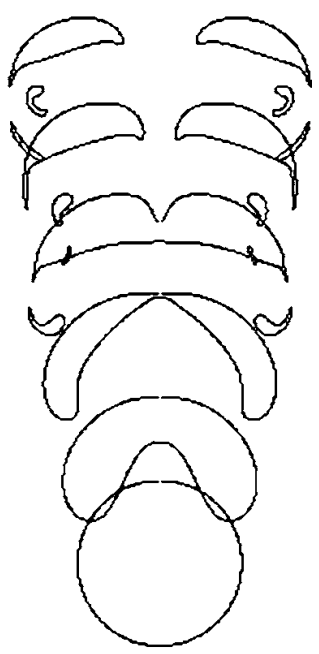

(b)
FIG. 8. Evolution of the shape of two bubbles with slightly different Archimedes numbers and infinite Bond number (left: $\mathrm{Ar}=79$; right: $\mathrm{Ar}$ $=84$ ). The time interval between successive views is $\Delta \tau=1.107$.

back. In this highly curved region the local shear generates a very thin toroidal ligament which breaks up and is quickly entrapped in the wake. The central part of the bubble then flattens while broadening radially, and the bubble reaches a final "flat cap" shape with a nearly constant thickness. Note that in this final stage the central part of the front has recovered a slightly positive curvature, while it was slightly negative during the transient stage around $\tau=3.32$. The evolution is pretty similar when $\mathrm{Ar}=84$, except that during the rethickening of the central region, the negative curvature at the front becomes larger and cannot be reduced anymore by viscous and transient effects $(\tau=3.32)$. The corresponding downward flow then quickly reduces the bubble thickness along its vertical diameter, which eventually leads to pinch-off at $\tau$ $\approx 4.0$ (Fig. 9).

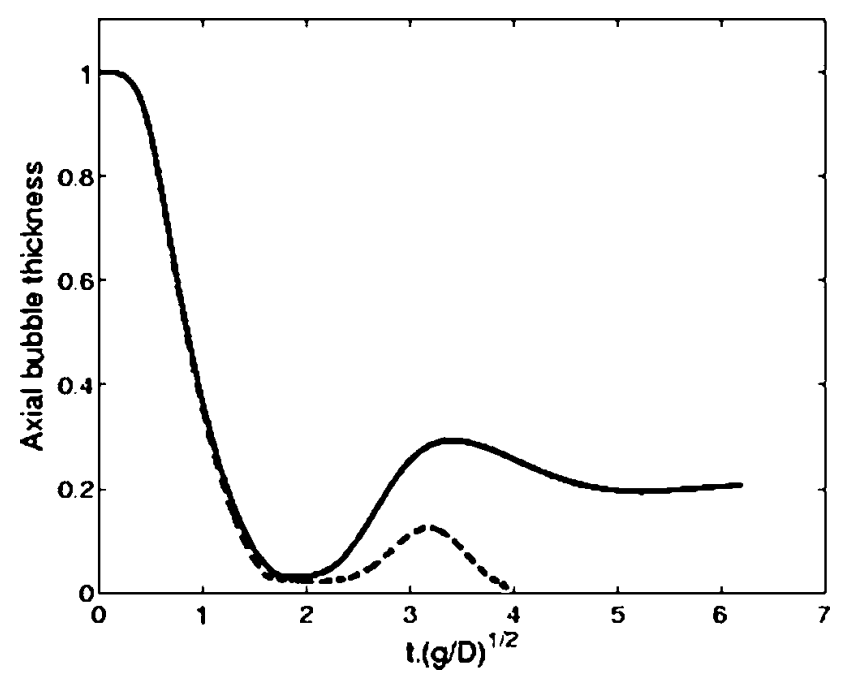

FIG. 9. Evolution of the bubble thickness along its vertical diameter (-) $(\mathrm{Bo}=\infty ; \mathrm{Ar}=79) ;(--)(\mathrm{Bo}=\infty ; \mathrm{Ar}=84)$. 


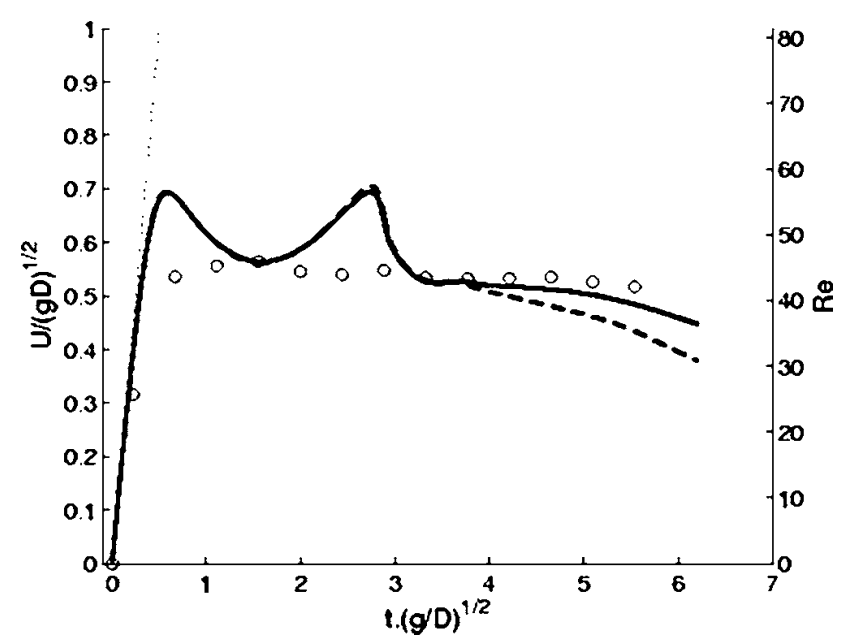

FIG. 10. Evolution of the bubble velocity and Reynolds number for two nearby Archimedes numbers $(\mathrm{Bo}=\infty)$. Open circles: front velocity for $\mathrm{Ar}$ =79; Solid line: centroid velocity for $\mathrm{Ar}=79$; dashed line: centroid velocity for $\mathrm{Ar}=84$; dotted line: $U=2 \mathrm{gt}$. The value of the Reynolds number on the right axis corresponds to $\mathrm{Ar}=81.5$

This viscous-inertial pinch-off mechanism appears to contrast with that prevailing in the high-Ar regime. In the latter regime, the front part of the bubble does not deform much and is pierced by the jet born in the rear part. In contrast, in the high-Bo viscous regime, the rear part of the bubble is able to recover an almost flat shape after the initial liquid tongue has broadened due to viscous effects. Then, pinch-off occurs because this rear part is pierced by a jet coming from the front region where the absence of surface tension makes the interface unable to sustain the overpressure produced by the local divergence of the streamlines. We are not aware of any previous description of this second formation scenario of toroidal bubbles. However, Collins ${ }^{26}$ noticed similar features for large two-dimensional bubbles which he observed to break along their centerline owing to a downward jet resulting from the instability of the concave bubble front.

Figure 10 shows the evolution of the bubble velocity in this high-Bo viscous regime. Again, the velocity of the bubble front first grows according to the inviscid prediction $U=2 g t$. Then, for $\mathrm{Ar}=79$, it stabilizes itself around $0.55(g D)^{1 / 2}$. The evolution of the centroid velocity is very similar for the two bubbles until pinch-off occurs at $\tau \approx 4.0$ for $\mathrm{Ar}=84$. Two maxima are observed in this evolution. The first of them $(\tau \approx 0.6)$ is due to the upward motion in the jet at the rear of the bubble, while the thickening of the central region at the expense of the external zone is responsible for the second maximum $(\tau \approx 2.8)$. It may be observed that the centroid velocity corresponding to this second maximum slightly increases by $1.8 \%$ from $\mathrm{Ar}=79$ to $\mathrm{Ar}=84$, owing to the increased strength of transient effects. This tiny difference is probably responsible for the ultimate pinch-off of the bubble in the latter case. During the late stage, the thin skirt of the bubble corresponding to $\mathrm{Ar}=79$ breaks up and is entrained in the wake, which results in a decrease of the centroid velocity. A sharper decrease, due to the impeding effect
TABLE I. Critical values of the Bond and Archimedes numbers for which the transition to a toroidal shape occurs, starting from a spherical shape.

\begin{tabular}{ccc}
\hline \hline & $\begin{array}{c}\text { Spherical cap } \\
\text { bubble }\end{array}$ & $\begin{array}{c}\text { Toroidal } \\
\text { bubble }\end{array}$ \\
\hline $\begin{array}{c}\text { Lundgren and Mansour } \\
\text { (Ref. 1) }\end{array}$ & $\mathrm{Bo}=32 ; \mathrm{Ar}=\infty$ & $\mathrm{Bo}=40 ; \mathrm{Ar}=\infty$ \\
(a) & $\mathrm{Bo}=32 ; \mathrm{Ar}=1.4 \times 10^{3}$ & $\mathrm{Bo}=35 ; \mathrm{Ar}=1.4 \times 10^{3}$ \\
(b) & $\mathrm{Bo}=35 ; \mathrm{Ar}=1.04 \times 10^{3}$ & $\mathrm{Bo}=35 ; \mathrm{Ar}=1.05 \times 10^{3}$ \\
(c) & $\mathrm{Bo}=50 ; \mathrm{Ar}=4.3 \times 10^{2}$ & $\mathrm{Bo}=50 ; \mathrm{Ar}=4.4 \times 10^{2}$ \\
(d) & $\mathrm{Bo}=10^{2} ; \mathrm{Ar}=1.8 \times 10^{2}$ & $\mathrm{Bo}=10^{2} ; \mathrm{Ar}=1.9 \times 10^{2}$ \\
(e) & $\mathrm{Bo}=3 \times 10^{2} ; \mathrm{Ar}=84$ & $\mathrm{Bo}=3 \times 10^{2} ; \mathrm{Ar}=90$ \\
(f) & $\mathrm{Bo}=10^{3} ; \mathrm{Ar}=79$ & $\mathrm{Bo}=10^{3} ; \mathrm{Ar}=84$ \\
Purely viscous regime & $\mathrm{Bo}=\infty ; \mathrm{Ar}=79$ & $\mathrm{Bo}=\infty ; \mathrm{Ar}=84$ \\
\hline \hline
\end{tabular}

of the circulation round the bubble core, is observed for the bubble corresponding to $\mathrm{Ar}=84$ after it has become toroidal.

\section{Intermediate regimes}

We also carried out a series of finite-Bond number computations in which Bo was varied from 32 to $10^{3}$. For each value of $\mathrm{Bo}$, we sought the critical value of the Archimedes number for which the transition to a toroidal shape occurs. These values are reported in Table I and the conclusions are displayed graphically in Fig. 11. Roughly speaking, the results indicate that the critical Bond number becomes insensitive to the precise value of the Archimedes number for $\mathrm{Ar} \geqslant 10^{3}$ (a regime which may be qualified as "purely capillary"), while the critical Archimedes number is almost independent of the Bond number for $\mathrm{Bo}>3 \times 10^{2}$, defining a "purely viscous" regime. The transition observed in the intermediate range is thus quite sharp, as it only covers roughly one decade in Bo and Ar. Note that in this intermediate regime, Chen et al. ${ }^{9}$ found that an initially spherical bubble

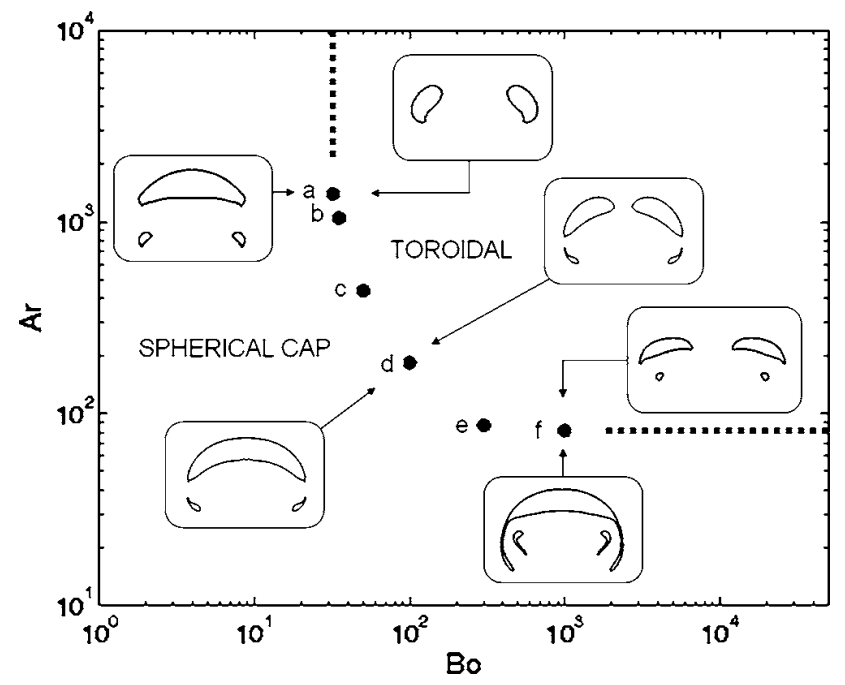

FIG. 11. Phase diagram of the transition from spherical cap bubbles to toroidal bubbles. The bubble shapes are those observed just below and above the transition in the late stages. Vertical and horizontal dashed lines indicate the location of the transition for the inviscid regime $(\operatorname{Ar}=\infty)$ and the purely viscous regime $(\mathrm{Bo}=\infty)$, respectively. 
corresponding to $\mathrm{Bo}=80$ and $\mathrm{Ar}=79$ (in our units) becomes toroidal, whereas Fig. 11 indicates that the transitional Archimedes number corresponding to $\mathrm{Bo}=80$ is beyond 200 . Examination of the computational parameters reveals that the grid used in Ref. 9 is 3.3 times coarser than in the present computations, and that the outer radius of the computational domain is only about 1 bubble diameter. In line with the tests reported in Fig. 3, we suspect that these computations are under-resolved and probably contaminated by confinement effects. Hence, we believe the discrepancy between our predictions and those of Ref. 9 concerning the position of the topological transition in the intermediate regime to be another illustration of the sensitivity of the numerical evolution of large buoyancy-driven bubbles to grid characteristics.

An important question concerns the comparison between present predictions for the position of the topological transition in the $(\mathrm{Bo}, \mathrm{Ar})$ plane and experimental data. For instance, Davies and Taylor ${ }^{10}$ report observations of spherical cap bubbles of $200 \mathrm{~cm}^{3}$ rising in water with a speed about $0.6 \mathrm{~m} / \mathrm{s}$. Using present definitions, the corresponding Bond and Archimedes numbers are 720 and $6.1 \times 10^{4}$, respectively, while the Reynolds number $\operatorname{Re}=\rho U D / \mu_{l}$ is about 4.35 $\times 10^{4}$. Hence, experiments indicate that, for a given Bond number, spherical cap bubbles may exist for Archimedes numbers at least two to three orders of magnitude larger than the transitional values indicated in Fig. 11. The obvious cause of this difference is the small-scale turbulence in the wake of such large-Re bubbles whose effect is not accounted for in present axisymmetric computations. We guess that this turbulence has a stabilizing effect on the rear part of the bubble as it tends to broaden the upward jet as if the liquid were locally very viscous. In contrast, as there is no turbulence upwards, the front part of the bubble experiences a purely capillary-inertial dynamics for such high values of Ar. Hence, the picture that emerges is that of a front part which evolves as we found numerically in the purely capillary regime, while the rear part evolves as in the purely viscous regime. No downward jet produced at the front of the bubble is then to be expected, while the upward jet coming from the back is considerably softened by the turbulent motion. Therefore, it is not surprising that spherical cap bubbles with a turbulent wake have a much larger domain of existence than predicted by present laminar computations. We can qualitatively confirm this point by the following reasoning. Let us assume that the effect of wake turbulence on the largescale flow at the rear of the bubble can be represented through an eddy viscosity $\mu_{e}$, even though the detailed physical relevance of the eddy viscosity concept may certainly be questioned. Following Prandtl's mixing length theory, we have $\mu_{e}=\rho \ell_{m}^{2} \bar{S}$, where $\ell_{m}$ is the corresponding mixing length and $\bar{S}$ is the characteristic strain rate of the time-averaged flow in the wake. This strain rate is dominated by the radial velocity gradient whose typical magnitude in the near wake is $2 U / D$, as the time-averaged velocity varies roughly from the rise velocity $U$ on the wake axis to zero beside the bubble. As the mixing length $\ell_{m}$ is generally considered to be about $0.1 D$ in free shear flows (meaning that there are typically ten large eddies across the wake), we ob- tain $\mu_{e} / \mu_{l} \approx 2 \times 10^{-2}$ Re. Defining an effective Archimedes number characterizing the relative momentum diffusion at the rear of the bubble as $\operatorname{Ar}_{e}=\rho_{l} g^{1 / 2} D^{3 / 2} / \mu_{e}$, we then have $\mathrm{Ar}_{e} / \mathrm{Ar}=\mu_{l} / \mu_{e} \approx 50 \mathrm{Re}^{-1}$. With $\mathrm{Re}=4.35 \times 10^{4}$, we finally estimate $\operatorname{Ar}_{e} \approx 70$. Hence, while the front of the bubble "sees" a very large Archimedes number Ar in the range $10^{4}-10^{5}$, momentum diffusion in the rear part corresponds to a much lower effective Archimedes number $\operatorname{Ar}_{e}$ of $O\left(10^{2}\right)$. Based on Figs. 4(a) and 8(a), we then expect the final shape at the front to be almost spherical while the rear should be almost flat. This is indeed what is seen in experimental photographs. ${ }^{12,15,16}$

\section{INFLUENCE OF INITIAL CONDITIONS}

As mentioned in Sec. I, available experimental results ${ }^{3,10}$ suggest that generating conditions play a crucial role in the topological evolution of large gas bubbles. Davies and Taylor ${ }^{10}$ notice that "considerable difficulty was found in producing single, large bubbles of gas, and the method finally adopted was to pivot an inverted beaker containing air, which was then tilted so that the air was released." This device enabled them to obtain spherical cap bubbles with a volume up to $200 \mathrm{~cm}^{3}$. In contrast, the system used by Walters and Davidson ${ }^{3}$ to generate toroidal bubbles in the same range of size consisted of introducing and entrapping air in a tube closed by a valve; the valve was then rapidly opened to release the bubble in the tank. That a given volume of air may give rise to two dramatically different bubbles topologies depending on the initial conditions deserves some more investigation. Note in particular that such a sensitivity to initial conditions is not observed with millimetric spheroidal bubbles rising in water. This point was examined by Yang et al., ${ }^{27}$ who performed axisymmetric computations of the evolution of such bubbles starting from various initial shapes. They found that, in all cases, bubbles of a given volume relax towards the same final oblate spheroidal shape.

To get some insight into this influence of initial conditions, we carried out a series of computations in which the initial bubble was given an oblate shape with an aspect ratio $\chi$ ranging from 1 to 3 ( $\chi$ is defined as the ratio of the major axis length to the minor axis length). The underlying idea is that the device used by Davies and Taylor produced fairly flat bubbles, whereas the photographs reported by Walters and Davidson indicate that their device created bubbles with a more or less spherical shape. A typical example of the results we obtained is shown in Fig. 12. In this case the Bond and Archimedes numbers are set to 100 and 400, respectively. According to Fig. 11, the critical value of Ar for Bo $=100$ is about 185 for an initially spherical bubble, so that $\mathrm{Ar}=400$ clearly corresponds to supercritical conditions. The conclusions revealed by Fig. 12 are clear: while the initially spherical bubble quickly becomes toroidal, the two initially oblate bubbles remain simply connected (except for a small secondary ring produced by the breakup of the tip during the stage where the bubble is skirted) and eventually reach a spherical cap shape. It is also worth noting that the latter two bubbles reach the same final shape. In dynamical system terms, what is suggested by Fig. 12 is that given a set 


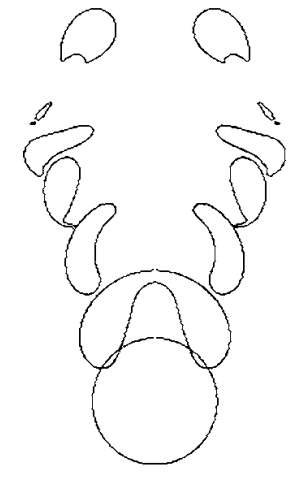

(a)

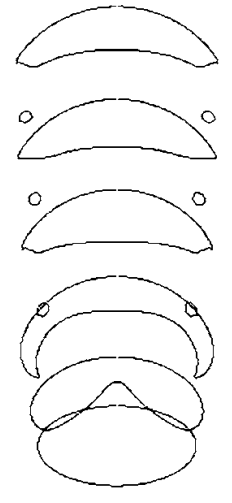

(b)

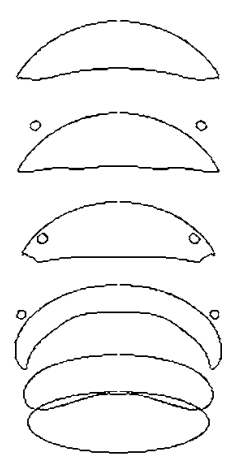

(c)
FIG. 12. Evolution of a bubble in the regime $\mathrm{Bo}=100, \mathrm{Ar}=400$ starting from (a) a spherical shape; (b) an oblate shape with an aspect ratio $\chi=2$; (c) an oblate shape with an aspect ratio $\chi=3$. The time interval between successive views is $\Delta \tau=1.11$.

(Bo, Ar) corresponding to supercritical conditions, the system has two stable fixed points (the toroidal shape and the spherical cap shape) with two well-defined basins of attraction characterized by two different ranges of the initial aspect ratio $\chi$.

We run several other cases, starting from nearly critical conditions given by Fig. 11, and increasing either the Bond number or the Archimedes number. These runs led to similar conclusions. For instance, with $\mathrm{Ar}=1.4 \times 10^{3}$, we observed that a bubble with an initial oblateness $\chi=2$ becomes toroidal only beyond $\mathrm{Bo} \approx 45$. We also run fully threedimensional computations to make sure that what we observe in Fig. 12 is not induced by the assumption of axisymmetry. These computations (whose spatial resolution is obviously less than that of the axisymmetric cases) merely produced the same results. In particular, we always found that oblate bubbles eventually turn into spherical caps under slightly supercritical conditions.

To better understand the crucial role of the initial shape in the bubble evolution, two physical mechanisms must be considered. First, the length of the minor (vertical) diameter of an oblate body evolves as $D \chi^{-2 / 3}$, which implies that the hydrostatic pressure difference between its two poles decreases as $\chi^{-2 / 3}$. Hence, the strength of the jet that forms at the rear of the bubble is a decreasing function of the oblateness. Second, an oblate body displaces more fluid than a sphere, and hence has a smaller acceleration when it starts to rise from rest. More precisely, the acceleration of a massless bubble rising parallel to one of its principal axes is $g / C_{M}$, where $C_{M}$ is the translational added-mass coefficient along the axis under consideration. The added-mass coefficient along the minor axis is ${ }^{28} C_{M}=\chi^{2}\left(\alpha-\operatorname{tg}^{-1} \alpha\right) /\left(\chi^{2} \operatorname{tg}^{-1} \alpha-\alpha\right)$, with $\alpha=\left(\chi^{2}-1\right)^{1 / 2}$, so that inertia effects resulting from bubble acceleration are about 3.5 times smaller for $\chi=3$ than for $\chi=1$. This implies that the Bond and Archimedes numbers based on $g / C_{M}$ (which, during the transient stages of the motion, are more meaningful than those based on $g$ alone) are decreasing functions of the initial oblateness, which indicates that the stability of the system increases with $\chi$.

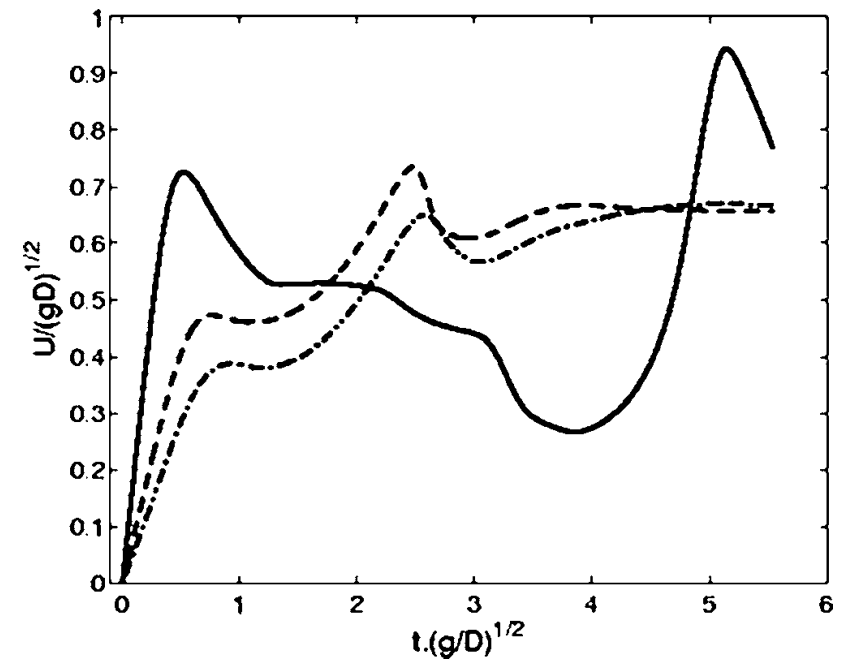

FIG. 13. Evolution of the centroid velocity, starting from: (a) - a spherical shape; (b) --- an oblate shape with $\chi=2$; (c) -...-. an oblate shape with $\chi$ $=3(\mathrm{Bo}=100 ; \mathrm{Ar}=400)$.

The influence of the initial oblateness on the bubble acceleration may easily be appreciated in Fig. 13. For $t(g / D)^{1 / 2} \leqslant 1.0$, which corresponds to the transient stage during which the upward jet develops, the two oblate bubbles rise significantly more slowly than the initially spherical bubble. This makes them capable of resisting more efficiently to the jet, thanks to the (relatively) stronger stabilizing influence of capillary and viscous forces. Note also on the second snapshot in Fig. 12 how the jet broadens and weakens as the oblateness increases, a direct consequence of the $\chi^{-2 / 3}$ decrease of the pressure difference between the two poles. Two other interesting features may be observed in Fig. 13. First, the rise velocity of the toroidal bubble is seen to undergo a huge increase for $4.0 \leqslant \tau \leqslant 5.0$. This is due to the reduction of the ring radius caused by the highly nonlinear shape oscillations visible in the left column of Fig. 12. This nonmonotonic evolution of the ring radius may be compared with the much more regular evolution displayed in Fig. 4(b) for $\mathrm{Bo}=35$. What this comparison reveals is that an increase of the Bond number by a factor of 3 is accompanied by a dramatic increase of the amplitude of core shape oscillations, which in turns affects the evolution of the rise velocity of the ring. Figure 13 also indicates that the two oblate bubbles eventually reach the same rise velocity, which confirms that they are relaxing towards the same final state, even though they had different initial oblatenesses. Their final radius of curvature $R_{c}$ is almost equal to $D$, so that their final rise velocity is very close to $0.67\left(g R_{c}\right)^{1 / 2}$, in agreement with the theoretical prediction of Davies and Taylor.

\section{SUMMARY}

We carried out a numerical investigation of the formation of gravitationally-driven toroidal bubbles in the presence of both capillary and viscous effects. For this purpose we used an original numerical method which solves the full Navier-Stokes equations on a fixed grid without involving an explicit reconstruction of the interface. We first checked that 
this method provides reliable predictions of the shape and velocity of bubbles rising in contrasted flow regimes and carefully examined the influence of the grid resolution on the predicted evolution of large buoyancy-driven bubbles. Based on these tests we stress again that the topological transition from spherical cap to toroidal bubbles is extremely sensitive to grid resolution, and that the proper description of the dynamics of these bubbles requires large resolutions.

After these preliminary steps, we examined in detail the conditions under which initially spherical bubbles may eventually become toroidal. We identified two different transition scenarios, corresponding to the limit of large Archimedes number (i.e., negligible viscous effects) and large Bond number (i.e., negligible capillary effects), respectively. In agreement with previous studies, we found the transition taking place in the capillary limit to be driven by an upward liquid jet that forms at the rear of the bubble, owing to the hydrostatic pressure difference existing between the two poles of the bubble. If surface tension is unable to limit the upward velocity, this jet eventually pierces the bubble front, yielding a toroidal bubble. We found this transition to take place in the range $32<\mathrm{Bo}<35$, in agreement with previous results obtained via the boundary element technique. ${ }^{1}$ The transition mechanism turns out to be different in the purely viscous limit. In this case we observed that a similar, however broader, jet forms at the rear but does not succeed to pierce the front. However, owing to the absence of surface tension, the front of the bubble can sustain the local pressure maximum only if viscous effects are sufficiently large. If not, the front becomes concave, forming a downward jet which pierces the rear of the bubble. We found this second type of transition to take place in the range $79<\mathrm{Ar}<84$. We also explored intermediate situations in which surface tension and viscosity both play a role, so as to localize the transition for arbitrary values of Bo and Ar. Corresponding results indicate that the critical Archimedes number decreases roughly from $10^{3}$ to $10^{2}$ when the Bond number increases from 35 to 300 . This contrasts with experimental results which report the existence of spherical cap bubbles with Bond and Archimedes numbers of $O\left(10^{2}\right)$ and $O\left(10^{4}\right)$, respectively. We attributed this difference to the wake turbulence which is not accounted for in the present axisymmetric computations and has a stabilizing influence on the rear part of the bubble, as it broadens and weakens the upward jet. By a crude estimate of the corresponding eddy viscosity, we showed that the Archimedes number based on the eddy viscosity, which is relevant to the dynamics of the rear part of the bubble, is only of $O\left(10^{2}\right)$. Hence, these bubbles appear to evolve in a very stable mixed regime as their rear part obeys a viscous dynamics (and is thus almost flat according to our numerical results), while the front part obeys a purely capillary dynamics (and is almost spherical).

We finally examined the influence of the initial shape on the final bubble topology by considering bubbles with various initial oblatenesses. We found that the bubble evolution depends dramatically on this initial condition, owing to the reduction of both the hydrostatic pressure difference between the two poles and the initial bubble acceleration as the oblateness increases. Since both effects contribute to reinforce the stabilizing influence of surface tension and viscosity, initially oblate bubbles may remain simply connected over a much wider range of Bond and Archimedes numbers than an initially spherical bubble of similar volume.

Obviously, the main limitation of the present study is related to the assumption of axisymmetry. Based on some fully three-dimensional computations we carried out, we do not believe the possible azimuthal shape instabilities not accounted for here modify dramatically the bubble evolution. In contrast, capturing the three-dimensional wake dynamics is of crucial importance to understand the possible influence of the wake on the bubble path (i.e., the generation of horizontal components of the bubble centroid velocity) as well as the stabilizing influence of the turbulent wake on the shape of the rear of the bubble. With this in mind, it is clearly desirable to develop such three-dimensional computations with a spatial resolution guaranteeing grid-independent results.

\section{ACKNOWLEDGMENTS}

Part of this work was supported by a research grant from Arcelor Research, whose help is very much appreciated. Some of the computational time was provided by the Scientific Groupment CALMIP where computations were run on Silicon Graphics Altix 3700 computers. We thank Richard Adoua for having provided the results concerning the rise of the oblate bubble obtained via the boundary-fitted version of JADIM in Sec. III. We also thank Annaig Pedrono for her constant and fruitful support in the improvement of the performances of the computer code.

${ }^{1}$ T. S. Lundgren and N. N. Mansour, "Vortex ring bubbles," J. Fluid Mech. 224, 177 (1991).

${ }^{2}$ K. Marten, K. Shariff, S. Psarakos, and D. J. White, "Ring bubbles of dolphins," Sci. Am. (Int. Ed.) 275, 64 (1996).

${ }^{3}$ J. K. Walters and J. F. Davidson, "The initial motion of a gas bubble formed in a inviscid liquid. Part 2. The three-dimensional bubble and the toroidal bubble," J. Fluid Mech. 17, 321 (1963).

${ }^{4}$ J. R. Blake and D. C. Gibson, "Cavitation bubbles near boundaries," Annu. Rev. Fluid Mech. 19, 99 (1987).

${ }^{5}$ J. P. Best, "The formation of toroidal bubbles upon the collapse of transient cavities," J. Fluid Mech. 251, 79 (1993).

${ }^{6}$ T. J. Pedley, "The toroidal bubble," J. Fluid Mech. 32, 97 (1968)

${ }^{7}$ J. S. Turner, "Buoyant vortex rings," Proc. R. Soc. London, Ser. A 239, 61 (1957).

${ }^{8}$ M. Sussman and P. Smereka, "Axisymmetric free boundary problems," J. Fluid Mech. 341, 269 (1997).

${ }^{9}$ L. Chen, S. V. Garimella, J. A. Reizes, and E. Leonardi, "The development of a bubble rising in a viscous liquid," J. Fluid Mech. 38, 61 (1999).

${ }^{10}$ R. M. Davies and G. I. Taylor, "The mechanism of large bubbles rising through extended liquids and through liquids in tubes," Proc. R. Soc. London, Ser. A 200, 375 (1950).

${ }^{11}$ J. F. Harper, "The motion of bubble and drops through liquids," Adv. Appl. Mech. 12, 59 (1972).

${ }^{12}$ P. P. Weneger and J.-Y. Parlange, "Spherical-cap bubbles," Annu. Rev. Fluid Mech. 5, 79 (1973).

${ }^{13}$ J. G. Hnat and J. D. Buckmaster, "Spherical cap bubbles and skirt formation," Phys. Fluids 19, 182 (1976); see also Erratum, ibid. 19, 611 (1976).

${ }^{14}$ R. Clift, J. R. Grace and M. E. Weber, Bubbles, Drops and Particles (Academic, New York, 1978).

${ }^{15} \mathrm{~T}$. Maxworthy, "A note on the existence of wakes behind large, rising bubbles," J. Fluid Mech. 27, 367 (1967)

${ }^{16}$ P. P. Weneger, R. E. Sundell, and J.-Y. Parlange, "Spherical cap bubbles rising in liquids," Z. Flugwiss. 19, 347 (1971).

${ }^{17} \mathrm{U}$. Brackbill, D. B. Kothe, and C. Zemach, "A continuum method for modeling surface tension,” J. Comput. Phys. 100, 335 (1992). 
${ }^{18}$ J. Magnaudet, M. Rivero, and J. Fabre, "Accelerated flows around a rigid sphere or a spherical bubble. I. Steady straining flow," J. Fluid Mech. 284, 97 (1995).

${ }^{19}$ I. Calmet and J. Magnaudet "Large eddy simulation of high-Schmidtnumber mass transfer in a turbulent channel flow," Phys. Fluids 9, 438 (1997).

${ }^{20} \mathrm{D}$. Legendre and J. Magnaudet, "The lift force on a spherical bubble in a viscous linear shear flow," J. Fluid Mech. 368, 81 (1998).

${ }^{21}$ S. T. Zalesak "Fully multidimensional flux-corrected transport algorithms for fluids," J. Comput. Phys. 31, 335 (1979).

${ }^{22}$ T. Bonometti, "Développement d'une méthode de simulation d'écoulements à bulles et à gouttes," Ph.D. dissertation, INPT, Toulouse, 2005.

${ }^{23}$ P. C. Duineveld, "The rise velocity and shape of bubbles in pure water at high Reynolds number," J. Fluid Mech. 292, 325 (1995).

${ }^{24} \mathrm{~A}$. Blanco and J. Magnaudet, "The structure of the axisymmetric high Reynolds number flow around an ellipsoidal bubble of fixed shape," Phys. Fluids 7, 1265 (1995).

${ }^{25}$ D. Legendre, J. Magnaudet, and G. Mougin, "Hydrodynamic interactions between two spherical bubbles rising side by side in a viscous liquid," J. Fluid Mech. 497, 133 (2003).

${ }^{26} \mathrm{R}$. Collins, "Structure and behaviours of wakes behind two-dimensional air bubbles in water," Chem. Eng. Sci. 20, 851 (1965).

${ }^{27}$ B. Yang, A. Prosperetti, and S. Takagi, "The transient rise of a bubble subject to shape or volume changes," Phys. Fluids 15, 2640 (2003).

${ }^{28}$ H. Lamb, Hydrodynamics, 6th ed. (Cambridge University Press, Cambridge, 1932). 